Although the cytotoxic mechanisms of the two drugs differ, both can trigger apoptosis as the result of their cytotoxic actions. We proposed that selection for resistance to either melphalan or doxorubicin would lead to defects in apoptotic signaling and execution. We used complementary DNA microarrays to evaluate the relative expression of approximately 5,700 human genes in a human multiple myeloma cell line (8226/S) and its melphalan-resistant subline (8226/LR5). We then compared the results to those from a previous study of doxorubicin resistance. Overall, $1-2 \%$ of the 5,700 genes analyzed were differentially expressed by a factor of two or more. Several genes were found to have altered expression resulting from selection with either doxorubicin or melphalan. Genes affected by selection with either drug included those encoding N1 spermine/spermidine acetyltransferase, hydroxysteroid (11- $\beta$ ) dehydrogenase, paraoxonase and TNF receptorassociated factor 3 . In addition, melphalan selection altered the same pathways as doxorubicin by affecting the expression of genes involved in apoptosis, such as those for Fas/CD95 and the anti-apoptotic BCL2-related protein A1. We conclude that melphalan and doxorubicin selection affected a common set of genes despite their differing mechanisms of action. These observations support apoptosis as a common endpoint for the cytotoxic actions of many chemotherapeutic drugs and indicate some of the points of the apoptotic process that can be altered by drug selection.

Weisz, Alessandro [60]

\section{Gene expression monitoring in hormone- responsive human breast cancer cells during estrogen-induced cell cycle progression}

Luigi Cicatiello ${ }^{1}$, Angelo Facchiano ${ }^{2}$, Raffaele Calogero ${ }^{3}$, Michele De Bortoli ${ }^{3}$, Francesco Bresciani ${ }^{1} \&$ Alessandro Weisz ${ }^{1}$

${ }^{1}$ Istituto Patologia generale e Oncologia, Seconda Università degli Studi di Napoli, Naples, Italy

${ }^{2}$ Centro di Ricerca Interdipartimentale di Scienze Computazionali e

Biotecnologiche, Seconda Università degli Studi di Napoli, Naples, Italy

${ }^{3}$ Università di Torino, Turin, Italy

Estrogens are potent mitogens for a subset of their target tissues, including those in breast cancer, in which they promote growth by interacting with specific intracellular receptors (ER- $\alpha$ and $-\beta$ ) of the nuclear receptor superfamily of transcription factors. Definition of the gene expression patterns, or signatures, characteristic of estrogen in growth-responsive cells would allow identification of the gene networks that mediate the cellular responses to these hormones and the discovery of new hormone-responsive genes and gene products that could be used as markers in experimental and clinical settings. For this purpose, we performed an initial characterization of the gene responses of human breast cancer cells to estrogen by determining the gene expression signatures of these cells before and after hormonal stimulation. We used microarrays containing more than 9,000 unique gene probes, about half of them unnamed. About $40 \%$ of the genes analyzed, including close to 1,200 expressed sequence tags, were detectable in RNA extracted from human breast cancer cells. Furthermore, the concentration of $10 \%$ messenger RNAs was either increased or decreased in the cell at one time or another after hormonal stimulation, each such change corresponding to a specific cell cycle phase. These included transcripts from known estrogen-responsive genes (such as $p S 2$, cfos, c-myc, and $c y c l i n D 1$ ) as well as a significant number of new hormonal targets. Hierarchical clustering shows distinct patterns of gene activation and inhibition, which will be exploited to further investigate intracellular signaling by steroid hormones in human breast cancer cells.
Westbrook, Carol

[61]

\section{A CDNA data set for expression studies of hematopoietic stem cells: characterization and database mining}

\author{
Carol Westbrook, Ignatius Gomes, Tiffany Le, Jeffrey Kapp \& \\ Ronald Hoffman
}

University of Illinois, Chicago, Illinois

Bone marrow cells that express the CD34+ antigen (CD34+ cells) represent a continuum of early and committed progenitor cells, including the most primitive stem cells. We used gene filter arrays to develop a complementary DNA database that is representative of the $\mathrm{CD} 34+$ transcriptosome, by comparing expression between human and baboon CD34+ cells to identify the most conserved and highly abundant genes in both species. RNA-based probes were sequentially hybridized to GeneFilters (Research Genetics) containing 25,920 human UniGene cDNAs. The human cells displayed 15,970 (62\%) of the cDNAs on the filters, of which $97 \%$ varied less than threefold in expression level between species and $60 \%$ were expressed sequence tags. Polymerase chain reaction analysis with reverse transcription confirmed that selected genes were expressed at the predicted levels. To demonstrate the utility of this stem cell database for gene discovery, we mined it for transcriptional regulators by searching UniGene cluster descriptors for text strings corresponding to relevant protein motifs, including transcription factor, zinc-finger, POU, homeobox, helix-loop-helix and leucine zipper. By this method we selected $230 \mathrm{cDNAs}$, of which 186 were known genes, including 71 transcription factors, and the remaining 44 were expressed sequence tags. This preliminary description of transcriptional regulators expressed in CD34+ cells provides a potential wealth of new information about the molecular basis of normal hematopoietic cell proliferation and demonstrates the utility of the stem cell database for gene discovery. This database should prove useful for the preparation of a rationally designed cDNA array for expression analysis of normal hematopoiesis, stem cells and leukemias.

Williams, Kathleen

[62]

\section{Patent strategies and patent disputes: SNPs, diseases, and treatment}

\section{Kathleen Williams}

Palmer \& Dodge, LLP, One Beacon Street, Boston, Massachusetts 02108, USA

Genome research and cancer treatment have presented challenges in defining what is meaningful within the large amounts of information generated in genomics and proteomics research. U.S. patent laws are in the process of clarifying what is patentable in genomics, gene expression, proteomics and bioinformatics. All these fields are the subject of intense patent activity, from patent disputes to the creation of patent strategies for protecting new technologies. What legal challenges might an investigator face as a discoverer of a technology that is key to disease treatment, whether it be a gene sequence, a mutation, a protein sequence or a method of dovetailing information on sequences and function? The law hands the researcher a sword to find legal avenues through patents and a shield to protect newly developed technologies. How can one effectively use these legal tools? 